\title{
LOS DERECHOS A LA TRADUCCIÓN Y A LA INTERPRETACIÓN RECONOCIDOS A LAS VÍCTIMAS EN EL PROCESO PENAL ESPAÑOL: LUCES Y SOMBRAS
}

\author{
Clara FERnÁndeZ CARRON \\ Profesora Contratada Doctora Interina \\ Departamento de Derecho Procesal \\ y Derecho Penal Facultad de Derecho \\ Universidad Complutense de Madrid \\ cfc@der.ucm.es
}

\begin{abstract}
RESUMEN
El presente trabajo tiene por objeto analizar el reconocimiento legal a la víctima alófona de su derecho a la traducción y a la interpretación en el marco del proceso penal. Se estudia el alcance con el que se han visto reconocidos ambos derechos a las víctimas y se van analizando las ventajas e inconvenientes que presenta la regulación legal en este punto, para acabar concluyendo que si bien esta es, en general, positiva, sin embargo, adolece de un problema importante: en la práctica, el ejercicio efectivo de ambos derechos por parte de la víctima alófona puede llegar a resultar muy difícil.
\end{abstract}

Palabras clave: víctima, derecho a la traducción, derecho a la interpretación, videoconferencia.

\section{ABSTRACT}

The current piece of work aims at studying the legal case of the victim to their right to Translation and Interpreting within the Penal procedure. We study the scope to which both rights have been foreseen and we study the advantages and disadvantages that a legal case shows to this purpose. We finish off by concluding that even if the latter is well thought, it lacks a clear point, however: in practice the effectiveness of both rights on the part of the victim may actually turn quite difficult.

Keywords: victim, right to translation, right to interpreting, videoconference.

\section{ZUSAMMENFASSUNG}

Die vorliegende Arbeit hat die rechtliche Anerkennung des anderssprachigen Opfers auf Übersetzung und Dolmetschleistungen im Rabmen des Strafprozesses zum Gegenstand. Es wird untersucht, wie weit bereits beide Rechte den Opfern zuerkannt wurden und es werden die Vor- und Nachteile der diesbezüglichen aktuellen gesetzlichen Regelung untersucht. Die Untersuchung kommt zu dem Schluss, 
dass die Bilanz positiv ist, jedoch zeigt sie auf, dass die derzeitige Regelung einen wichtigen Mangel erleidet: in der Praxis kann die Ausübung des Rechts für das andersprachige Opfer sebr schwierig verlaufen.

Schlüsselwörter: opfer, recht auf übersetzung, recht auf dolmetschen, videokonferenz.

SUMARIO: I. OBSERVACIONES PRELIMINARES.-II. ORIGEN DE LA LEVD.III. DERECHO A LA TRADUCCIÓN Y DERECHO A LA INTERPRETACIÓN RECONOCIDOS A LAS VÍCTIMAS.-1. Cuestiones generales.-2. Derecho a la interpretación.-3. Derecho a la traducción.-IV. CONCLUSIONES.

\section{OBSERVACIONES PRELIMINARES}

La finalidad del presente estudio radica en el análisis de un punto muy preciso del régimen jurídico de la víctima en el proceso penal español: el reconocimiento por parte del legislador de los derechos a la traducción y a la interpretación. Habida cuenta de la extensión de este trabajo, procederemos en primer lugar a abordar brevemente el análisis del origen de la Ley 4/2015, de 27 de abril, del Estatuto de la Víctima del Delito (en adelante, LEVD), que entre el catálogo general de derechos comunes que reconoce a todas las víctimas regula los derechos a la traducción e interpretación reconocidos a las víctimas del delito ${ }^{1}, \mathrm{y}$ que fue desarrollada posteriormente por el Real Decreto 1109/2015, de 11 de diciembre, por el que se desarrolla la LEVD y se regulan las Oficinas de Asistencia a las Víctimas del Delito (en adelante, RD 1109/2015). A continuación centraremos nuestra atención en determinar el alcance del derecho a la traducción y del derecho a la interpretación, y, finalmente, concretaremos las ventajas e inconvenientes que, a nuestro juicio, presenta la regulación legal de ambos derechos.

\section{ORIGEN DE LA LEVD}

El primer precedente del «Estatuto de la víctima» lo constituye la Decisión Marco 2001/220/JAI del Consejo, de 15 de marzo de 2001, relativa

${ }^{1}$ En este punto queremos llamar la atención sobre el hecho de que esta ley debería haber sido orgánica y no una ley ordinaria como así lo ha sido, dado que afecta a derechos fundamentales de la víctima, con lo que se vulnera lo dispuesto en el art. 81.1 CE. 
al estatuto de la víctima en el proceso penal (en adelante, DM), que reconoce un conjunto de derechos de las víctimas en ese ámbito. Aunque esta, con la que se pretendía lograr un reconocimiento homogéneo y uniformador de la víctima en el ámbito de la UE, no fue desarrollada por los países de la Unión, sin embargo, algunos sí que publicaron su normativa específica. Esto es lo que ocurrió, entre otros, con nuestro Estado, en el que, aunque con un ámbito y alcance diferentes, se aprobaron algunas normas que atendían a colectivos concretos de víctimas de determinados delitos (terrorismo, libertad sexual, violencia de género, etc.), pero que no ofrecían un catálogo sistemático y unificado de derechos de la víctima, y que, por tanto, no brindaban una respuesta conjunta a la situación de la víctima en general ni tampoco, en particular, a su situación ante el proceso penal ${ }^{2}$. Centrándonos ya en el origen de la LEVD, lo primero que hay que advertir es que en el Anteproyecto de esta Ley (en adelante, ALEVD), el legislador pretendió introducir la trasposición de dos Directivas: de un lado, la Directiva 2010/64/UE del Parlamento Europeo y del Consejo, de 20 de octubre de 2010, relativa al derecho a interpretación y a traducción en los procesos penales (en adelante, DIT), y de otro, la Directiva 2012/29/UE del Parlamento Europeo y del Consejo, de 25 de octubre de 2012, por la que se establecen normas mínimas sobre los derechos, el apoyo y la protección de las víctimas de delitos (en adelante, DPVD), que sustituyó a la DM. Dado que la opción de introducir en la LEVD la trasposición de la DIT fue rápidamente rechazada ${ }^{3}$, la LEVD, en síntesis, y por lo que ahora interesa, vino a transponer a nuestro Derecho interno la DPVD ${ }^{4}$.

${ }^{2}$ Lo que ocurrió, entre otras, con la Ley 35/1995, de 11 de diciembre, de Ayudas y Asistencia a las Víctimas de Delitos Violentos y contra la Libertad Sexual (desarrollada por el RD 738/1997, de 23 de mayo); la Ley Orgánica 1/1996, de 15 de enero, de Protección Jurídica del Menor; la Ley Orgánica 1/2004, de 28 de diciembre, de Medidas de Protección Integral contra la Violencia de Género; la Ley 29/2011, de 22 de septiembre, de Reconocimiento y Protección Integral a las Víctimas del Terrorismo, y la Ley Orgánica 1/1996, de 15 de enero, de Protección Jurídica del Menor.

${ }^{3}$ Finalmente, tal Directiva se traspuso a nuestro ordenamiento jurídico a través de la Ley Orgánica 5/2015, de 27 de abril, por la que se modifican la Ley de Enjuiciamiento Criminal y la Ley Orgánica 6/1985, de 1 de julio, del Poder Judicial, para transponer la Directiva 2010/64/UE, de 20 de octubre de 2010, relativa al derecho a interpretación y traducción en los procesos penales, y la Directiva 2012/13/UE, de 22 de mayo de 2012, relativa al derecho a la información en los procesos penales (en adelante, LO 5/2015).

${ }^{4}$ Sobre este particular téngase presente que, tal y como así se señala en el punto II del Preámbulo de la LEVD, «el presente texto legislativo no solo responde a la exigencia de mínimos que fija el legislador europeo con el texto finalmente aprobado en la citada Directiva 2012/29/UE, sino que trata de ser más ambicioso, trasladando al mismo las demandas y necesidades de la sociedad española, en aras a completar el diseño del Estado de Derecho, 


\section{DERECHO A LA TRADUCCIÓN Y DERECHO A LA INTERPRETACIÓN RECONOCIDOS A LAS VÍCTIMAS}

\section{Cuestiones generales}

Antes de adentrarnos en el análisis de los derechos a la traducción y a la interpretación reconocidos a las víctimas de delitos deben tenerse presentes las siguientes consideraciones previas:

a) La aprobación de la LEVD no ha supuesto la derogación de la normativa nacional especial existente en nuestro ordenamiento jurídico destinada a reconocer los derechos de los colectivos de víctimas que presentan especiales necesidades o una mayor vulnerabilidad. De ello se deduce que, por lo que respecta al reconocimiento de los derechos a la traducción y a la interpretación, habrá de estarse a lo dispuesto en toda esta normativa, conjugando las previsiones que se recojan al respecto en cada una de ellas.

b) La LEVD ha supuesto un cambio radical en la forma de entender la participación de la víctima en el proceso judicial. Por fin está presente en el proceso y debe ser tenida en cuenta desde su inicio, reconociéndosele una participación mucho más activa en él y, de solicitarlo, debiendo ser informada cumplidamente del estado de la causa durante su tramitación, o al menos de sus hitos más relevantes, llegando incluso a permitirla recurrir determinadas resoluciones aun cuando no esté previamente personada $y$, en ocasiones, incluso sin necesidad de asistencia letrada. En consecuencia, la LEVD ha incrementado notablemente las exigencias derivadas del derecho a interpretación y a traducción.

c) A la hora de reconocer derechos a las víctimas - directas o indirectas (art. 2 LEVD)—, la LEVD no diferencia entre la gravedad del delito. Esto se corrobora con la lectura de su art. 1, en el que se dispone que las disposiciones de la ley serán aplicables a las víctimas de delitos cometidos en España o que puedan ser perseguidos en España. Así, se han de reconocer los mismos derechos a las víctimas de delitos leves que a las que lo sean

centrado casi siempre en las garantías procesales y los derechos del imputado, acusado, procesado o condenado [...]. Con ese foco de atención se ha podido advertir, y así lo traslada nuestra sociedad con sus demandas, una cierta postración de los derechos y especiales necesidades de las víctimas del delito que, en atención al valor superior de justicia que informa nuestro orden constitucional, es necesario abordar, siendo oportuno hacerlo precisamente con motivo de dicha transposición». 
de delitos graves, cosa que, atendiendo al importante número de derechos que se les reconoce en la LEVD y teniendo en cuenta la escasez de recursos humanos y materiales, conduce a que, en la práctica, resulte muy difícil alcanzar la efectividad real de tales derechos, por lo que hubiera sido muy positivo que, en aras a priorizar y asignar más eficientemente la escasez de recursos, la LEVD hubiera diferenciado a las víctimas atendiendo a criterios de mayor o menor gravedad del delito.

d) Los derechos que reconoce la LEVD a las víctimas (arts. 3 y ss.) tienen un ámbito de aplicación temporal bastante extenso: se les garantiza su ejercicio antes del comienzo del proceso (es decir, desde el primer contacto con autoridades o funcionarios), a lo largo de todo el proceso y tras su conclusión, durante un periodo de tiempo adecuado.

e) El primero de los derechos básicos de las víctimas, denominado «derecho a entender y ser entendida», está relacionado directamente con garantizar la comunicación; condición previa e indispensable para asegurar la efectividad de su derecho a participar en el proceso. Tal derecho se reconoce en su máxima extensión, ya que es exigible en todo tipo de actuaciones que tengan que ver con el delito cometido - no ya solo desde la interposición de la denuncia y durante el proceso penal, sino incluso con carácter previo a la interposición de aquella - y ya sea en sede administrativa, policial o judicial. A fin de que el derecho de la víctima a entender y ser entendida en cualquier actuación pueda ejercitarse plenamente, el art. 4 LEVD señala que todas las comunicaciones con las víctimas, orales o escritas, se han de hacer en un lenguaje claro, sencillo y accesible, teniéndose en cuenta las características personales de aquellas y, especialmente, las necesidades de las personas con discapacidad sensorial, intelectual o mental o su minoría de edad.

e) Una vez garantizada la comunicación, puede desplegar ya toda su eficacia el derecho a la información reconocido a las víctimas tanto desde el primer contacto que tengan con las autoridades competentes, incluyéndose aquí también el momento previo a la interposición de la denuncia (art. 5 LEVD), como sobre la causa penal (art. 7 LEVD). Por lo que respecta a lo primero, el art. 5 LEVD, en el que se recoge un extenso elenco de derechos que habrán de comunicarse a la víctima, señala que toda víctima tiene derecho a recibir, sin retrasos innecesarios, información adaptada a sus circunstancias y condiciones personales y a la naturaleza del delito cometido y de los daños y perjuicios sufridos, sobre una serie de extremos entre los que, por lo que a nosotros ahora interesa, se incluye la información sobre los servicios de interpretación y traducción disponi- 
bles $^{5}$, además de la información relativa al derecho a efectuar una solicitud para ser notificada de ciertas resoluciones ${ }^{6}$.

f) Aunque la DPVD, al igual que lo hizo la DIT, reconoce de forma independiente dos derechos distintos, el derecho a interpretación (art. 7.1) y el derecho a traducción (art. 7.3), distinción que, a nuestro juicio, resulta muy acertada porque con ella se favorece la visibilidad de ambos derechos, se clarifica su alcance y se refuerza el contenido sustancial de cada uno de ellos ${ }^{7}$, la LEVD se ha decantado por regular de forma entremezclada ambos derechos, regulándolos de forma conjunta en su art. 9 rubricado «Derecho a la traducción e interpretación». Pues bien, en este punto radi$\mathrm{ca}$, a nuestro juicio, uno de los principales inconvenientes que presenta la técnica legislativa empleada en la LEVD: aunque se trata de dos derechos que presentan aspectos comunes, son distintos e independientes el uno del otro, exigiéndose habilidades y competencias diferentes para el ejercicio de cada uno de ellos, con lo que regularlos de forma entremezclada a lo único a lo que coadyuva, desgraciadamente, es a aumentar la confusión que, a día de hoy, reina ya desde hace tiempo respecto de las profesiones de traductor e intérprete incluso en el ámbito de nuestros Tribunales.

5 En este punto es importante destacar que, a diferencia de lo que ocurre con el derecho a interpretación y a traducción reconocidos al sospechoso o acusado alófono en los arts. 123 a 127 LECrim., en los que se prevén mecanismos para reaccionar frente a la falta de calidad tanto de la interpretación como de la traducción, entendida en el sentido de que no ofrezcan «garantías suficientes de exactitud», lo que se explica muy bien en la STS de 26 de enero de 2016 (entre otros, la posible sustitución del intérprete o del traductor y la creación de registros oficiales de traductores e intérpretes debidamente cualificados), la LEVD no contiene ninguna previsión respecto de las exigencias del nivel de tales derechos reconocidos a la víctima, por lo que la efectividad de tales derechos puede llegar a quedar en entredicho, en la medida en que no se ha previsto expresamente un mecanismo de sustitución del traductor o intérprete en caso de que sus servicios hubieran resultado deficientes.

${ }^{6}$ El deber de información a la víctima de sus derechos y de los extremos a que se refiere la LEVD en su art. 5 corresponde a todas las autoridades, funcionarios y Oficinas de Asistencia a las Víctimas del Delito, que habrán de hacerlo de oficio. En un primer momento puede corresponder a la policía, al Ministerio Fiscal y a las Oficinas de Asistencia y, una vez judicializado el asunto, se entiende a cargo fundamentalmente de los letrados de la Administración de Justicia.

7 Con ello, la DPVD se desmarca de instrumentos anteriores tales como el Convenio Europeo de Derechos Humanos, el Pacto Internacional de Derechos Civiles y Políticos, el Estatuto de Roma, la Carta de Naciones Unidas de San Francisco de 1945 o la Declaración Universal de Derechos Humanos de 1950, entre muchos otros, en los que el derecho a la asistencia lingüística se refiere esencialmente al derecho a interpretación, quedando el derecho a la traducción a la sombra de aquel, no habiéndose visto reconocido hasta un momento posterior [el nacimiento del derecho a la traducción puede concretarse en el momento en que el Tribunal Europeo de Derechos Humanos interpretó lo dispuesto en el art. 6, $\mathbb{\$} 3$, a) y $e$ ), $\mathrm{CEDH}]$. 
g) La LEVD guarda absoluto silencio respecto del plazo para proceder tanto a la traducción de los documentos como a la asistencia de intérprete, a diferencia de lo que ocurre con la LECrim. en la que, respecto al derecho a traducción reconocido a los sospechosos y acusados, se señala que habrá de hacerse en «un plazo razonable». Aunque si bien ante la ausencia de previsión legal al respecto habrá que entender que la traducción y/o la provisión de un intérprete a la víctima alófona habrá de llevarse a cabo lo antes posible, tal solución, no obstante, plantea importantes problemas por cuanto con ella no parece posible determinar a partir de qué momento podrá la víctima impugnar que se ha producido una vulneración en su derecho a la traducción o a la interpretación.

\section{Derecho a la interpretación}

El art. 9 LEVD reconoce una serie de derechos a toda víctima que no hable o no entienda el castellano o la lengua oficial que se utilice en la actuación de que se trate ${ }^{8}$; previsión esta última relativa a la lengua oficial que no se recogía en el ALEVD? .

Entre ellos, y centrándonos ahora en el derecho relativo a la interpretación, tal precepto dispone que la víctima alófona tendrá derecho a ser asistida gratuitamente por un intérprete que hable una lengua que comprenda cuando se le reciba declaración en la fase de investigación por el juez, el fiscal o funcionarios de policía, o cuando intervenga como testigo en el juicio o en cualquier otra vista oral, derecho también aplicable a las perso-

${ }^{8}$ Con la expresión de «víctima que no hable o no entienda el castellano o la lengua oficial que se utilice en la actuación de que se trate», el legislador de la LEVD ha sido mucho más atinado que el legislador de la LO 5/2015. Este último, al redactar el nuevo art. 123 LECrim., dedicado a la regulación del derecho a la traducción y a la interpretación, reconoce tales derechos a los imputados o acusados que «no hablen o entiendan el castellano o la lengua oficial en la que se desarrolle la actuación»; expresión que, atendiendo a su tenor literal, podría llevar al absurdo de reconocer tales derechos a quienes entiendan el castellano o la lengua oficial en la que se desarrolle la actuación, cosa que, sin embargo, atendiendo al tenor del art. 9 LEVD, no puede ocurrir.

${ }^{9}$ La inclusión de la previsión sobre la lengua oficial en la LEVD se debió a haberse acogido una de las previsiones recogidas en la conclusión vigésimo séptima del Informe emitido por el CGPJ al AEVD, de fecha 31 de enero de 2014, como era el hecho de que, en atención a la cooficialidad lingüística de algunas Comunidades Autónomas, debía hacerse referencia también a la lengua cooficial que pudiera utilizarse o en la que se desarrollara el proceso (el contenido del Informe puede consultarse en el siguiente enlace: $h t t p: / / w w w$ poderjudicial. es/cgpj/es/Poder-Judicial/Consejo-General-del-Poder-Judicial/Actividad-del-CGPJ/Informes/ Informe-alAnteproyecto-de-Ley-Organica-del-Estatuto-de-las-Victimas-del-delito). 
nas con limitaciones auditivas o de expresión oral ${ }^{10}$. Sobre este particular señalar que, aunque el art. 21.b) LEVD, con el fin de evitar en la medida de lo posible la victimización secundaria, señala que se recibirá declaración a la víctima el menor número de veces posible y únicamente cuando resulte estrictamente necesario, vista la estructura de nuestro proceso penal —en la práctica, la víctima presta varias veces declaración (ante la policía, la ratifica en el Juzgado, el Ministerio Fiscal suele pedirle otra en fase de instrucción y en juicio oral) - habrá de reconocérsele el derecho a interpretación en todas estas declaraciones. Posteriormente, el art. 9.2 LEVD señala que la asistencia de intérprete se podrá prestar por medio de videoconferencia o cualquier medio de telecomunicación, salvo que el juez o tribunal, de oficio o a instancia de parte, acuerde la presencia física del intérprete para salvaguardar los derechos de la víctima. Aunque esta posibilidad pueda estar pensada para evitar distorsiones por déficit de medios personales, se debería imponer como regla general la presencia física del intérprete junto a la víctima, convirtiendo el recurso a la videoconferencia en excepción, dado que estar presente virtualmente a través de esta no puede equipararse a la presencia física en ningún caso, ya que aquella presenta importantes problemas tanto a nivel de deficiencias o dificultades técnicas (de visión, de audición y generales, tales como las averías informáticas del propio sistema, etc.), como de condicionantes externos que afectan a las declaraciones, como, finalmente, presenta el serio inconveniente de que su uso conduce a una profunda deshumanización de la justicia, lo que se hace especialmente patente cuando de la víctima de un delito se trata, dada la situación de especial vulnerabilidad en la que se encuentra. Aunque la interpretación a distancia o remota presenta ciertas ventajas ${ }^{11}$, nunca debería prevalecer en

${ }^{10}$ Aunque la LEVD no se refiere expresamente a qué técnica deberá utilizarse para llevar a cabo la interpretación, si la simultánea, en la que la interpretación se realiza en tiempo real y de forma paralela al discurso de quien habla, pues el intérprete traslada el mensaje del orador con un mínimo desfase temporal al oyente, o la consecutiva, en la que la interpretación se produce a posteriori una vez que el orador ha finalizado de hablar y después de que el intérprete haya tomado las notas correspondientes, la práctica demuestra que en sede policial suele utilizarse la modalidad de interpretación consecutiva, mientras que ante nuestros tribunales suele utilizarse la interpretación simultánea —cuando disponen de los equipos técnicos necesarios (cabinas cerradas, micrófonos, auriculares, etc.) - y cuando no dispongan de ellos, lo que ocurre en la mayoría de los casos, la interpretación consecutiva o la susurrada, subespecialidad de la interpretación simultánea que consiste en interpretar de forma simultánea en voz baja al oído de la víctima.

${ }^{11}$ La videoconferencia es un medio idóneo para cumplir los plazos del proceso sin que este se demore injustificadamente, ya que permite que, en caso de lenguas o dialectos minoritarios de escaso uso que hagan imposible encontrar un intérprete por razones de tiempo o 
detrimento de la presencia física del intérprete: sustituir la presencia física por la presencia a distancia debería ser excepcional, restringiéndose pues el uso de la videoconferencia en los casos en que resulte realmente justificada la absoluta imposibilidad de que el intérprete se desplace a tiempo al lugar donde se desarrollen las actuaciones. En definitiva, asumiendo un mal menor para evitar males mayores, entendemos que solo debería procederse al uso de la videoconferencia para facilitar la interpretación con una víctima cuando se constate la necesidad de recurrir a los servicios de interpretación para hacer efectiva la asistencia a la víctima sin demora y ello no pueda hacerse de otro modo, por resultar excesivo para aquella el tiempo de espera para poder proveerla de la presencia física del intérprete y ser, por tanto, en el caso concreto lesivo para sus derechos.

De otro lado, y aunque así no se recoja expresamente en la LEVD, parece lógico entender que a la víctima también ha de reconocérsele la asistencia lingüística a la hora de comunicarse con su abogado en los casos en que requiera de asistencia letrada, debiendo, por tanto, reconocérsele el derecho a interpretación para las comunicaciones orales que tenga con su abogado. A la víctima también se le reconoce el derecho a la asistencia lingüística gratuita a la hora de presentar su denuncia [art. 6.b)]. Igualmente, y dado que el art. 11.b) LEVD reconoce el derecho de la víctima a comparecer ante las autoridades encargadas de la investigación para aportarles las fuentes de prueba y la información que estime relevante para el esclarecimiento de los hechos ${ }^{12}$, habrá que entender que, en estos casos, deberá reconocérsele el derecho a ir acompañada de un intérprete, cosa que puede predicarse también para los casos en que la víctima interese que se impongan al liberado condicional las medidas o reglas de conducta previstas por la ley que consideren necesarias para garantizar su seguridad; desee facilitarle al tribunal cualquier información que resulte relevante para resolver sobre la ejecución de la pena impuesta (art. 13.2 LEVD), o bien haya solicitado información relativa a la situación o estado del procedimiento (art. 7.4 LEVD). Sobre este último particular, señalar que este derecho plantea un problema importante: resulta muy difícil determinar qué se entiende por informar y hasta dónde llega dicho deber de informa-

de distancia, se pueda contactar y acceder a interpretaciones de un gran abanico de idiomas o de combinaciones lingüísticas poco comunes en una amplia franja horaria.

${ }^{12} \mathrm{El}$ reconocimiento de tal derecho es fundamental, dado que, como señala la DPVD, «no se puede hacer justicia si no se permite a las víctimas explicar las circunstancias del delito y aportar pruebas de forma comprensible para las autoridades competentes» (considerando núm. 34). 
ción, en la medida en que la LEVD se refiere con carácter genérico a informar sobre «la situación en que se encuentra el procedimiento».

Finalmente, en los casos en que la víctima se halle ante actuaciones policiales, de denegársele su derecho a interpretación —-decisión policial que, a tenor de lo dispuesto en el art. 6 del RD 1109/2015, será excepcional y motivada, debiendo quedar debida constancia de la misma y de su motivación en el atestado que, a su vez, deberá recoger la disconformidad formulada por la víctima ante la decisión denegatoria-, el art. 9.4 LEVD le reconoce la posibilidad de recurrir tal decisión ante el juez de instrucción, entendiéndose interpuesto tal recurso cuando la víctima hubiera expresado su disconformidad en el momento de la denegación. Sobre este particular señalar simplemente que se trata de un recurso completamente nuevo y singular, inexistente hasta la fecha, sobre cuya tramitación poco se especifica en la LEVD más allá de su constancia en el atestado, pues ni tan siquiera se señala plazo para resolverlo. En cambio, si la decisión de no facilitar interpretación a la víctima fue judicial, a tenor de lo dispuesto en el art. 9.5 LEVD, podrá ser recurrida en apelación ${ }^{13}$.

\section{Derecho a la traducción}

El reconocimiento del derecho a la traducción procede solo cuando la víctima en su primera comparecencia haya solicitado la notificación de las resoluciones a que se refieren los arts. 7.1 y 12 LEVD [por remisión del art. 9.1.b) y c)]; comunicaciones que le serán remitidas a su dirección de correo electrónico o, excepcionalmente, cuando no disponga de ella, por correo ordinario a la dirección que hubiera facilitado ${ }^{14}$. La traducción de

13 Sobre este particular llama especialmente la atención el hecho de que el legislador de la LEVD no haya optado por establecer el mismo régimen de recursos en este punto que aquel por el que se ha decantado el legislador de la LO 5/2015 en los casos en que la denegación de la interpretación se produzca respecto del sospechoso o acusado, ya que este ha optado por remitir a estos últimos, cuando deseen recurrir la decisión denegatoria de la designación de un intérprete, al régimen general de recursos establecido en la LECrim. En este punto hemos de admitir que no logramos encontrar una justificación al hecho de que el legislador, a la hora de regular una misma cuestión —el régimen de recursos frente a las decisiones denegatorias del reconocimiento del derecho a interpretación-, establezca un régimen diferente en función de que quien se haya visto privado de este derecho sea un sospechoso o acusado o la víctima de un delito.

${ }_{14}$ De lo dicho se deduce claramente que: a) las notificaciones dependen de la voluntad de la víctima, que, además, puede renunciar a ellas en cualquier momento (art. 7.2 LEVD), de modo que el reconocimiento del derecho a traducción procede únicamente a demanda 
las resoluciones se circunscribe, al menos, a su parte dispositiva y a un breve resumen de los fundamentos, lo que supone una traducción parcelaria, cabiendo excepcionalmente sustituir la traducción escrita de documentos por un resumen en forma oral de su contenido en una lengua que comprenda la víctima, cuando de este modo se garantice suficientemente la equidad del proceso (art. 9.3 LEVD). Sobre este último particular señalar que si bien permitirse, aunque sea de forma excepcional ${ }^{15}$, sustituir la traducción escrita por un resumen en forma oral del contenido de los documentos es una facultad que tiene sus ventajas, puesto que conlleva un menor coste económico y puede ser preferible por razones de tiempo, ya que obtener una traducción oral inmediata y no esperar a una escrita evita dilaciones temporales, sin embargo, tal previsión restringe en cierta medida el derecho a la traducción.

La víctima tiene también derecho a que se le entregue una copia de la denuncia traducida [art. 6.a) LEVD]; a que se le informe en una lengua que comprenda de la fecha, hora y lugar de celebración del juicio [art. 9.1.d) LEVD], así como del contenido de la acusación dirigida contra el infractor (art. 7.1 LEVD), información que, aunque no se establece expresamente en tales preceptos que deba realizarse de forma escrita, habrá que entender que así habrá de hacerse ${ }^{16}$; a la traducción gratuita de los extremos sobre los que debe ser informada a tenor de lo dispuesto en el art. 5 LEVD, para lo que en principio se cuenta ya con impresos o formularios de diligencias de información de tales derechos traducidos a los

del justiciable alófono, a diferencia de lo que ocurre con el sospechoso o acusado a quien, además de a su instancia, deberá reconocérsele el derecho a traducción e interpretación de oficio por parte de las autoridades o funcionarios competentes en cuanto se pongan de manifiesto circunstancias de las que pueda derivarse la necesidad de asistencia de un intérprete o traductor, y b) que la LEVD delimita las resoluciones a notificar, de modo que no se notifica cualquier resolución dictada en el procedimiento, sino solo las que indica la ley.

15 Adviértase en este punto que la LEVD no precisa qué ha de entenderse por «excepcionalmente», por lo que habrá que entender que la posibilidad de sustituir la traducción por un resumen en forma oral debe quedar circunscrita a los casos en que exista una razón de necesidad que haga imposible la espera de la traducción escrita.

${ }^{16}$ Lo que se deduce, a nuestro juicio, del hecho de que el art. 785.3 LECrim. señala que cuando la víctima lo haya solicitado, el secretario judicial deberá informarle, por escrito y sin retrasos innecesarios, de la fecha, hora y lugar del juicio, así como del contenido de la acusación dirigida contra el infractor. No obstante lo dicho, y atendiendo a lo dispuesto en el art. 27 del RD 1109/2015, también cabría entender que tal información podrá realizarse de forma oral, en cuyo caso entraríamos en el ámbito del derecho a la interpretación, dado que aquel precepto prevé que las Oficinas de Asistencia a las Víctimas darán la información que precisa la víctima adaptada a sus circunstancias y condiciones personales, a la naturaleza del delito cometido y a los daños y perjuicios sufridos; información que podrá ser por escrito, verbal o por medios electrónicos, así como presencial o no. 
idiomas más frecuentes ${ }^{17}$, y a la traducción gratuita de aquella información que resulte esencial para el ejercicio de los derechos a que se refiere el título II LEVD (todos ellos relativos a su participación activa en el proceso penal, incluida la fase de ejecución), pudiendo presentar a su vez una solicitud motivada para que se considere esencial un documento ${ }^{18}$ [art. 9.1.c) LEVD].

Pues bien, por lo que respecta a las concretas resoluciones que deberán ser traducidas, lo primero que hemos de señalar es que la LEVD ha sido muy generosa en este punto, incluyendo un importante número de resoluciones que habrán de notificarse a la víctima y que, por tanto, habrán de ser traducidas a una lengua que comprenda. Además de reconocérsele el derecho a obtener la traducción escrita de la copia de la denuncia presentada, lo que requerirá la disponibilidad inmediata de intérpretes, cosa que no siempre resultará posible, máxime en los casos en que la víctima hable una lengua o dialecto minoritario, habrán de notificárseles las siguientes resoluciones: la que acuerde no iniciar el procedimiento penal; la sentencia que ponga fin al procedimiento; las resoluciones que acuerden la prisión o la posterior puesta en libertad del infractor, así como la posible fuga del mismo; las resoluciones que acuerden la adopción de medidas cautelares personales o que modifiquen las ya acordadas; las resoluciones o decisiones de cualquier autoridad judicial o penitenciaria que afecten a sujetos condenados por delitos cometidos con violencia o intimidación y que supongan un riesgo para la seguridad de la víctima; las resoluciones a que se refiere el art. 13 (relativas todas ellas a determinados autos dictados por el juez de vigilancia penitenciaria) ${ }^{19}$, y la resolución de sobreseimiento.

Finalmente, por lo que respecta al régimen de recursos ante la decisión denegatoria del derecho a traducción, se trata del mismo que el dispuesto para la denegación del derecho a interpretación, por lo que, para evitar reiteraciones innecesarias, véase lo dicho al respecto en el epígrafe anterior.

17 Por citar algunos, inglés, alemán, francés, ruso, chino, rumano y árabe. Téngase presente en este punto que, de no tratarse de lenguas frecuentes, difícilmente podrá cumplirse el mandato del art. 5 LEVD.

${ }^{18}$ Previsión esta última que, a nuestro juicio, aunque si bien tiene todo su sentido cuando se refiere al derecho a traducción de los documentos que se consideren esenciales reconocido al sospechoso o acusado, dada la incidencia que ello puede tener respecto de la salvaguarda de su derecho de defensa, ya que permite que se le pueda garantizar estar en condiciones de ejercerlo, cobra menos sentido en caso de la víctima.

19 Sobre este particular llama poderosamente la atención el hecho de que la víctima sí tenga derecho a la traducción de las resoluciones que se dictan en fase de ejecución y que, por el contrario, la LO 5/2015 no haya reconocido tal derecho al penado. 


\section{CONCLUSIONES}

Aunque si bien es cierto que, gracias a la aprobación de la LEVD, en los últimos tiempos se han reforzado en nuestro ordenamiento jurídico los derechos y garantías reconocidos a las víctimas del delito, hemos de admitir que, sin restar valor a lo que la LEVD les supone a estas últimas, dado que han tenido que esperar más de un siglo desde la publicación de la LECrim. para ver reconocidos sus derechos, más cierto aún es el hecho de que muchos de estos resultan inoperativos en la práctica, dada la carencia de medios y recursos personales. Por lo que a nosotros ahora intere$\mathrm{sa}$, esto es lo que ocurre precisamente con los preceptos de la LEVD que reconocen los derechos a la traducción y a la interpretación a las víctimas que, muy a nuestro pesar, en muchos casos no suponen más que meras declaraciones de intenciones. Y ello es así porque, teniendo en cuenta, de un lado, lo previsto en la disposición adicional segunda de la LEVD, en la que se señala que «las medidas incluidas en la misma no podrán suponer incremento de dotaciones de personal ni de retribuciones $u$ otros gastos de personal», y, de otro, que los derechos a interpretación y traducción a la víctima se han reconocido en la LEVD ampliamente, de modo que las exigencias derivadas de la interpretación de diligencias orales y de la traducción de actuaciones escritas van a verse notablemente incrementadas, tal disposición adicional deja en papel mojado las previsiones de la LEVD relativas a ambos derechos, ya que para su ejercicio efectivo en la práctica parece obvio que resulta imprescindible destinar más medios personales $y$, por tanto, incrementar el número de intérpretes y traductores. El reconocimiento y efectividad de los derechos a traducción y a interpretación que regula la LEVD no pueden pasar por su aplicación «a coste cero». Aunque en este punto el legislador aparenta ser muy garantista con la protección de tales derechos, la realidad es que en su intención subyace algo diametralmente opuesto: reconoce derechos «en vacío», ya que, sin medios, no puede existir un reconocimiento efectivo de los derechos a interpretación y traducción que, recuérdese, han de garantizarse a las víctimas de forma gratuita, con el incremento de costes económicos que ello entraña para las arcas públicas. 